activation of this enzyme, reported by GuTMAN et al. ${ }^{2,3}$. Although these authors state that thenoyltrifluoroacetone is without effect on the activation by NADH, their data (cf. Figs. 1 and 2 of ref. 3) show that, after $1 \mathrm{~min}$, the rate of activation by $\mathrm{NADH}$ is inhibited by $50 \%$. In view of the facts that thenoyltrifluoroacetone does not inhibit completely the flux of electrons between succinate dehydrogenase and the respiratory chain (see e.g. ref. 11) and that piericidin sufficient to inhibit

1 E. B. Kearney, J. biol. Chemistry 229, 363 [1957].

2 M. Gutman, E. B. Kearney, and T. P. Singer, Biochem. biophysic. Res. Commun. 42, 1016 [1971].

3 M. Gutman, E. B. Kearney, and T. P. Singer, Biochemistry 10, 2726 [1971].

4 T. Y. WAnG, C. L. Tsou, and Y. L. Wang, Sci. Sinica Peking 5, 73 [1956].

5 D. Keilin and T. E. King, Proy. Roy. Soc. London, Ser. B, 152, 163 [1960].

6 W. P. Zeylemaker, Succinaatdehydrogenase. Eigenschappen en reactiemechanisme, $\mathrm{Ph}$. D. thesis, Amsterdam, Mondeel, Amsterdam 1969.
NADH oxidation by $97 \%$ does not inhibit the activation ${ }^{3}$, the incomplete inhibition of the activation by thenoyltrifluoroacetone is readily explained. It is concluded that insufficient evidence has been provided for a specific role of ubiquinol in the activation of succinate dehydrogenase. However, the possible physiological role of $\mathrm{NADH}$ activation of succinate oxidation, suggested by GUTMAN et al. ${ }^{3}$, does not depend upon the specific mechanism proposed by these authors.

7 A. Kröger and M. Klingenberg, Biochem. Z. 344, 317 [1966].

8 T. E. King, R. L. How ARd, D. F. Wilson, and J. C. R. LI, J. biol. Chemistry 237, 2941 [1962].

9 T. E. King, Nature [London] 198, 366 [1963].

10 P. Cerletti, R. Strom, and M. G. Giordano, Arch. Biochem. Biophysics 101, 423 [1963].

11 S. P. J. Albracht, H. van Heerikhuizen, and E. C. Slater, Biochim. biophysica Acta [Amsterdam] 256, 1 [1972].

\title{
Sequence around the Active Center Cystine of Lipoamide Dehydrogenase from Pig Heart, Comparison with the E. coli Enzyme*
}

\author{
C. H. Williams, JR. and L. D. ArscotT \\ The Veterans Administration Hospital and The Department of Biological Chemistry, \\ The University of Michigan, Ann Arbor, Michigan 48105 \\ (Z. Naturforsch. 27 b, 1078-1080 [1972] ; received May 10, 1972)
}

Sequence, active center, lipoamide dehydrogenase, enzyme

\begin{abstract}
Catalysis by lipoamide dehydrogenase involves the concerted action of the flavin and a cystine residue. Peptides containing this cystine residue have been previously isolated from E. coli and now from pig heart. The sequences of amino acid residues reveal a high degree of homology indicating a strict conservation of the region around the active site cystine during the long evo. lutionary period between these two species. The peptide sequences suggest a likely conformation of the polypeptide chain in the region of the flavin as well as the forces involved in substrate and flavin binding.
\end{abstract}

The pyridine nucleotide-disulfide oxidoreductases, lipoamide dehydrogenase, thioredoxin reductase and glutathione reductase are catalytically similar flavoproteins characterized by the presence of a redox active cystine residue functioning together with the flavin in electron transfer ${ }^{1-4}$. We have recently reported the structures of peptides containing the active center cystine isolated from E.coli lipoamide dehydrogenase and thioredoxin reductase and the

Requests for reprints should be sent to C. H. Williams, JR., Gen. Med. Res., V. A. Hospital, 2215 Fuller Road, Ann Arbor, Mich. 48105, U.S.A. latter structure has also been reported by THelandeR ${ }^{5-8}$. Enzymes of this group contain a single cystine and three or more cysteine residues. (There is some evidence to suggest that pig heart lipoamide dehydrogenase may contain a second disulfide $^{9}$; but our data indicate that this may be artifactual.) Provided certain precautions are observed in order to prevent thiol-disulfide interchange, the isolation of peptides containing the active site

\footnotetext{
* This work was supported in part by Grant AM 09313 from the National Institute of Arthritis and Metabolic Diseases. U.S.P.H.S.
} 
cystine presents no problems. In the present communication, we report on the structure of an analogous peptide from pig heart lipoamide dehydrogenase and discuss some of the implications of these structures.

Pig heart lipoamide dehydrogenase is made up of two polypetide chains (identical or near identi$\mathrm{cal}^{6}$ ) with two moles of FAD tightly bound. The molecular weight is 102,000 . Each polypeptide chain contains 10 half-cystines (cystine plus cysteine) determined as cysteic acid following oxidation by dimethyl sulfoxide ${ }^{\mathbf{1 0}}$. The measurement of free thiols in the presence of $5 \mathrm{M}$ guanidine hydrochloride using fresh enzyme indicates the presence of 7 cysteines, 6.9 in repeated titrations with $5,5^{\prime}$-dithiobis-(2nitro-benzoic acid) and $6.4-6.9$ by reaction with iodoacetate and measurement of S-carboxymethylcysteine after hydrolysis. Thus the 7 cysteines plus the active center cystine do not account for the 10 half-cystines. At present our data do not permit us to distinguish between the several possible explanations of this anomaly.

The methods used in the isolation of the pig heart lipoamide dehydrogenase cystine peptide were essentially the same as those used previously ${ }^{5-7}$ and reflect the need to prevent thiol-disulfide interchange: reaction of the free thiols with maleimide; digestion with pepsin; and purification first on Sephadex G-25 and then by ion-exchange chromatography. The subtractive-Edman procedure was used for sequencing.

The sequence of the cystine containing peptide from pig heart lipoamide dehydrogenase is given below, together with the previously determined sequence of the analogous peptide from the E.coli enzyme ${ }^{6}$ aligned so as to display maximum homology.

\section{Pig heart}

$$
\text { Glu-Thr-Leu-Lys-Gly-Thr- Cys-Leu-Asn-Val-Gly-Cys-Ile-Pro-Ser-Ala-Ieu }
$$

E. coli

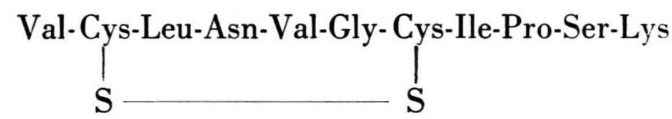

The following observations were made ${ }^{6}$ concerning the peptide isolated from the $E$. coli enzyme and are equally valid for this very similar peptide from the pig heart enzyme. The fact that only four amino acid residues are interposed between the two half-cystines makes it quite likely that the polypeptide chain assumes a rather flat conformation in this region. It should also be noted that most of the residues in this region are hydrophobic. It is tempting to speculate that the flat isoalloxazine portion of the FAD is bound to this flat portion of the polypeptide chain providing many possible sites of hydrophobic interaction. It has been previously suggested on the basis of the highly resolved spectrum of this enzyme that the flavin is bound in a hydrophobic region ${ }^{11}$. It has also been shown that there is a very close chemical interaction between the FAD and the disulfide during catalysis ${ }^{1}$, thus necessitating the very close spatial juxtaposition. This hydrophobic region must also be involved in the binding of the substrate, lipoamide.

Nine of 11 overlapping residues are identical. This is the longest homology thus far reported for analogous peptides isolated from enzymes of a prokaryote and eukaryotic mitrochondria. Thus this portion of the polypeptide chain has been rigidly conserved during the long period of evolution subsequent to the divergence of these two species from a presumed common ancestor. This rigid conservation is perhaps not surprising given the rather demanding catalytic requirements for close interaction, initially between lipoamide (covalently bound to the transacetylase) and the redox active disulfide; then between the disulfide and the flavin; and finally between the FAD-thiol complex and the pyridine nucleotide which may also be protein bound. 
1 V. Massey and C. Veeger, Biochim. biophysica Acta [Amsterdam] 48, 33 [1961].

2 V. Massey and C. H. Williams, JR., J. biol. Chemistry 240 , 4470 [1965].

3 G. Zanetti and C. H. Williams, JR., J. biol. Chemistry 242, 5232 [1967].

4 L. Thelander, Eur. J. Biochem. 4, 407 [1968].

5 C. H. Williams, JR., B. D. Burleigh, JR., S. Ronchi, L. D. ArscotT, and E. T. Jones, in: H. Kamin, editor, Flavins and Flavoproteins, Vol. III, p. 295, University Park Press, Baltimore, Md., 1971.
6 B. D. Burleigh, JR. and C. H. Williams, JR., J. biol. Chemistry 247, 2077 [1972].

7 S. Ronchi and C. H. Williams, JR., J. biol. Chemistry 247, 2083 [1972].

8 L. Thalander, J. biol. Chemistry 245, 6026 [1970].

9 G. Palmer and V. Massey, Biochim. biophysicsa Acta [Amsterdam] 58, 349 [1962].

10 R. L. Spencer and F. Wold, Anal. Biochem. 32, 185 [1969].

11 G. Palmer and V. Massey, in: T. P. Singer, editor, Biological Oxidations, p. 263, Interscience Publications, Inc., New York 1968.

\title{
Butyryl Coenzyme A Dehydrogenase: \\ Studies on the Specificity of the Formation of Acyl Coenzyme A Complexes with Long-Wavelength Absorption
}

\author{
P. C. ENGEL \\ Department of Biochemistry, University of Sheffield, Sheffield S10 2TN, England \\ (Z. Naturforsch. 27 b, 1080-1081 [1972] ; received May 10, 1972)
}

\begin{abstract}
Butyryl CoA dehydrogenase, acyl CoA, long-wavelength absorption, charge transfer complexes, alicyclic
\end{abstract}

\begin{abstract}
Further studies of butyryl CoA dehydrogenase show that the enzyme forms tight complexes with long-wavelength absorption bands upon addition of $\alpha, \beta$-unsaturated alicyclic carboxylic acyl CoA compounds. As in the straight chain series, the size of the acyl moiety is critical. The thiol portion by contrast determines neither whether a long-wavelength band is formed nor the wavelength of maximum absorption if such a band is present. It does however markedly affect the dis sociation constant. Unusual features of the acid stability of the acyl CoA found on the native green form of the enzyme are also reported.
\end{abstract}

It has been shown ${ }^{1}$ that green butyryl CoA dehydrogenase from Peptostreptococcus elsdenii is a complex between free enzyme and tightly bound acyl CoA. The uncomplexed yellow form of the enzyme can be prepared by anaerobic dialysis of chemically-reduced enzyme followed by reoxidation. Many $\alpha, \beta$-unsaturated and $\beta$-keto acyl CoA compounds form tight complexes with the yellow form of the enzyme and some of these complexes, like the native green enzyme, have long-wavelength absorption bands. The formation of such absorption bands and the wavelength of maximal long-wavelength absorption are both markedly dependent upon the chain length of the acyl moiety. For instance in the homologous series of straight-chain $\alpha, \beta$-unsaturated acyl CoA compounds only pent-2-enoyl CoA gives rise to a long-wavelength band $(810 \mathrm{~nm})$. If the acyl chain is either shorter or longer by one carbon

Requests for reprints should be sent to Dr. P. C. ENGEL, Department of Biochemistry, University of Sheffield, Sheffield S10 2TN, England. atom complexes are formed which show no longwavelength absorption.

These observations suggested that in the active site of the enzyme the acyl chain of the substrate or inhibitor may be curled up in a hydrophobic pocket of limited size. This would account for the chain length specificity of the enzyme. Accordingly the complexing ability of the CoA thioesters of alicyclic carboxylic acids has now been tested. Cyclopent-2-ene carboxylic acyl CoA and cyclobut-2-ene carboxylic acyl CoA both form tight complexes with long-wavelength absorption bands maximal at 620 and $710 \mathrm{~nm}$ respectively. The corresponding cyclohexene compound does not yield a long-wavelength band.

The specificity for the thiol portion of the molecule has also been examined by using the acetoacetyl thioesters of Coenzyme A, pantetheine, $\mathrm{N}$-acetylcysteamine and ethanethiol. These all form complexes with long-wavelength absorption bands centred at $580 \mathrm{~nm}$ upon addition to yellow butyryl 